\title{
Droplet Image Feedback Control System in Evaporation Experiment
}

\author{
Qiang Yu • Shijie Cai • Zhiqiang Zhu • Qiusheng Liu • \\ Binghong Zhou
}

Received: 15 February 2009 / Accepted: 10 August 2009 / Published online: 26 August 2009

(C) Springer Science + Business Media B.V. 2009

\begin{abstract}
In order to realize the steady-state droplet evaporation, image feedback control system is designed based on DSP. The system has three main functions: to capture and store droplet images during the experiment; to calculate droplet geometrical and physical parameters such as volume, surface area, surface tension and evaporation velocity at a high-precision level; to keep the droplet volume constant. The DSP can drive an injection controller with the PID control to inject liquid so as to keep the droplet volume constant. The evaporation velocity of droplet can be calculated by measuring the injected volume during the evaporation. The structure of hardware and software of the control system, key processing methods such as contour fitting and experimental results are described.
\end{abstract}

Keywords Image processing • Evaporation velocity • Steady-state evaporation

Q. Yu (凶) · S.-J. Cai · B.-H. Zhou

Center for Space Science and Applied Research, Chinese

Academy of Sciences, Beijing 100190, China

e-mail: yuqiang@cssar.ac.cn

S.-J. Cai

e-mail: caishijie06@gmail.com

B.-H. Zhou

e-mail: bhzhou@cssar.ac.cn

Z.-Q. Zhu • Q.-S. Liu

National Microgravity Laboratory, Institute of Mechanics,

Chinese Academy of Sciences, Beijing 100190, China

e-mail: zhuzhiqiang@imech.ac.cn

Q.-S. Liu

e-mail: liu@imech.ac.cn

\section{Introduction}

Steady-state evaporation experimental study is becoming a valuable approach to investigate the temperature jump at the interface, the coupling mechanism between thermocapillary convection and evaporation effect, and the contact angle etc. in recent years (Duan et al. 2005; Badam et al. 2007). Steady-state evaporation of a droplet under micro-gravity provides an especially ideal situation to study the above phenomenon (Zhu 2009).

The apparatus for investigation of evaporation is implemented to realize steady-state evaporation (Popov et al. 2005). The water level can be recorded with a cathetometer by visual observation of the water surface. By controlling the feed rate of water and the evaporation rate, the location of free water surface can be maintained constant throughout an experiment. However, this approach is inconvenient: the operator needs to observe the water level and adjust the feed rate of water manually to realize steady-state evaporation. Thus, it is not feasible for experiment on recoverable satellite where there is no operator. To overcome this shortage, an image feedback control system is designed to realize steady-state evaporation in droplet evaporation experiment for future Chinese recoverable satellite. Image processing technology is introduced within this control system to control the feed rate of liquid automatically. Surface tension, contact angle and other experimental parameters are measured at the same time.

There are numerous methods developed for the measurement of surface tension and contact angle of liquid (Rotenberg and Boruvka 1983; Bechstedt 2003; Erbil 2006; Hartland 2004). Taking advantage 
of new advances in image processing in recent years, the drop-shape method has been developed. Entropic edge-detection method is introduced to deal with the source image of the drop, which is affected by any kind of noise, blur or low-contrast effect (Atae-Allah et al. 2001). The entire axisymmetric drop shape analysis technique, including the hardware and software, has been reviewed (Hoorfar and Neumann 2004). These studies provide the theoretical bases and possible solutions to determine the surface tension and contact angle.

Droplet image feedback control system is a specialized fluid experimental equipment, which is developed for the evaporation and fluid interface effect experiment on recoverable satellite. This system not only keeps the droplet size unchanged during experiment, but also uses the image processing techniques to get real-time access to surface tension and contact angle. The droplet size and surface area can be calculated by numerical integration method; surface tension and contact angle can be determined by contour fitting. With the droplet volume real-time obtained, the droplet size is kept unchanged by adopting automatic control method. The evaporation rate can also be calculated by recording the volume of injection per unit time and unit surface area.

The paper is structured as follows. "Introduction" introduces the image feedback control system. "Structure of Droplet Image Feedback Control System" introduces the hardware and software structure of the system. "Droplet Contour Fitting Algorithm" reveals the droplet contour fitting algorithm and "Experimental Results" presents the experimental results.

\section{Structure of Droplet Image Feedback Control System}

\section{Hardware Structure}

The hardware structure of droplet image feedback control system is illustrated in Fig. 1 (Yu and Ning 2008). The system consists of a CCD camera, a DSP image

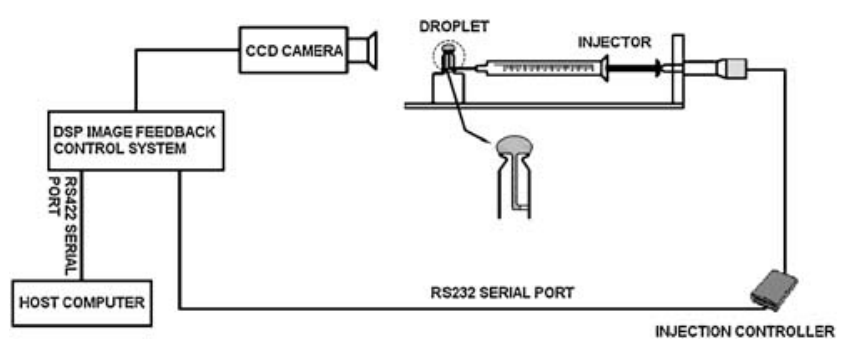

Fig. 1 Hardware structure of the system

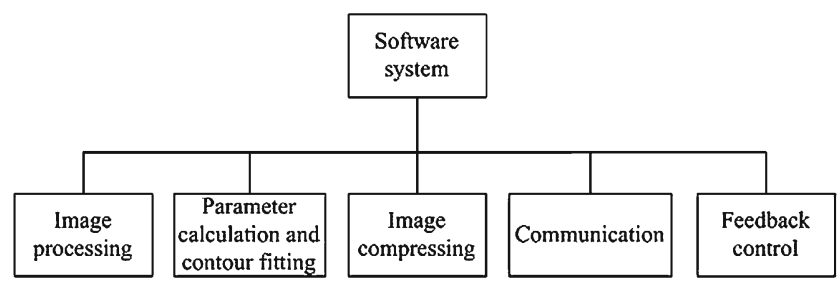

Fig. 2 Software structure of the droplet feedback control system

feedback control system, an injection controller and an injector, and droplet experiment platform. The CCD camera uses Toshiba TELI standard camera which is mounted with lens and the image resolution is $576 * 768$. The CCD camera is used to acquire image and output the image to the DSP image processor. The DSP image processor is the hardware to realize the feedback control algorithm. With DSP image processor, we can get the contour of droplet, realize the contour fitting and calculate parameters. The image is compressed and analyzed at the same time. The control signal is outputted to the injector. The images and key parameters are sent from the DSP image processor to the host computer. The injector is controlled by computer through serial port RS232, the flow is as low as $0.001 \mu \mathrm{l} / \mathrm{hr}$ and the precision is higher than $0.5 \%$.

\section{Software Structure}

The Software includes an image processing unit, a parameter calculation and a contour fitting unit, an image compression unit, a feedback control unit and a communication unit. The software structure of the droplet feedback control system is shown in Fig. 2.

The external contour of droplet is accessed through image processing on droplet. Figure 3 shows the droplet image processing in the experiment. The process is as follows:

First, use the Sobel gradient operator to detect the edge of the grayscale image (Gonzalez et al. 2003). Then the image is dealt with basic global thresholding (Weszka 1978). The following algo-

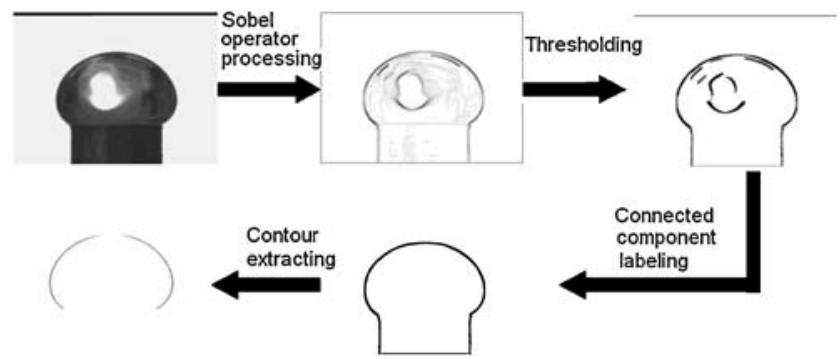

Fig. 3 The image pre-processing of a droplet experiment 
rithm can be used to obtain the threshold $T$ automatically:

1. Select an initial estimate for $T$.

2. Segment the image using $T$. This will produce two groups of pixels: $G_{1}$ consisting of all pixels with gray level values $>T$ and $G_{2}$ consisting of pixels with values $\leq T$.

3. Compute the average gray level values $\mu_{1}$ and $\mu_{2}$ for the pixels in regions $G_{1}$ and $G_{2}$.

4. Compute a new threshold value:

$$
T=\frac{1}{2}\left(\mu_{1}+\mu_{2}\right)
$$

5. Repeat steps 2 through 4 until the difference in $T$ in successive iterations is smaller than a predefined parameter $T_{o}$.

In order to improve the robustness of droplet image feedback control system, connected component labeling algorithm is applied to droplet contour extraction. It can distinguish every connected domain by marking it with different values, which represent different connected domain. By choosing the domain with largest area, the desirable domain will be extracted and the internal false contour and noise will be removed. A one-dimensional table is used to memorize label equivalences in the optimized algorism, which can speed up the processing speed (Suzuki et al. 2003). In order to realize a faster processing speed and smaller memory occupation, run-length encoding is introduced. Moreover, by changing the assignment of label connection table, only one scanning is needed to memorize all label equivalences in the processing (Cai and Yu 2008).

Finally, the external contour is extracted by scanning the desirable domain again to record coordinates of the extreme left and the extreme right pixels in every line.

Parameter calculation and contour fitting unit outputs the key parameters of the droplet (Ning and Zhu 2008). The surface area and volume of the droplet can be calculated by numerical integration of the droplet contour. Through contour fitting process, the surface tension and contact angle can be calculated. The error function is established which measures the differences between the calculated Laplacian curve and the measured curve. The minimization of the error function will determine the shape parameter $\beta$ and the radius of curvature $R_{0}$ at the origin of the $x-z$ coordinate system. Thus the surface tension and contact angle are also determined. The process will be elaborated in chapter 3 .

The image compression unit compresses the image losslessly so that to reduce the time of sending images. Because the time to deal with each frame is reduced, the interval of control system is shortened and the control quality is improved. The JPEG-LS algorithm is comparatively faster among the lossless compression methods, and higher compression rate can be reached (Weinberger et al. 2000). We adopted this algorism for compressing images and get a 4:1 compression ratio of the original.

Communication unit is the interface among DSP processor, host computer and injector. This unit can transmit compressed data of each image frame and the key parameters to the host computer so that we can save the processing results of each image frame. It can also send orders to the injector, and implement the automatic control of the injector operation.

Feedback control unit realizes the volume control of the droplet by calculating the volume of droplet and using PID control method to determine the inject amount of droplet.

\section{Droplet Contour Fitting Algorithm}

\section{Principle of the Droplet Contour Profile Fitting}

For any point $p$ on curving droplet surface, the inner pressure $P_{1}$ is not equal to the outer pressure $P_{2}$. The pressure difference $\Delta P=P_{1}-P_{2}$ is related to the droplet interface curvature and $\Delta P$ is formulated as follow:

$\Delta P=\gamma\left(\frac{1}{R_{1}}+\frac{1}{R_{2}}\right)$

This is the Laplace equation (Yuan et al. 2002) about droplet surface, in which $\gamma$ is surface tension; $R_{1}$ and $R_{2}$ is the first and the second curvature radius at the point $\mathrm{p}$ respectively.

If there is only one force - the gravity, pressure difference can be formulated as:

$\Delta P=\Delta P_{0}+(\Delta \rho) g z_{1}$

$\Delta P_{0}$ is the pressure difference at a selected datum plane, $\Delta \rho$ is the difference in the densities of the two bulk phases, $g$ is gravitational acceleration, $z_{1}$ is vertical height measured from the datum plane. $(\Delta \rho) g z_{1}$ is the hydrostatic fluid pressure difference. As shown in Fig. 4, the peak point in the curve is selected as the coordinates origin, axis $\mathrm{x}$ is a tangent line through the origin, $O_{1}$ and $O_{2}$ is the two centers of the circle of curvature passing the point $p$. Because of the symmetry properties, $R_{1}=R_{2}=R_{0}$, at the origin. So $\Delta P=2 \gamma / R_{0}$.

Because $R_{2}=x_{1} / \sin \phi$, Eqs. 1 and 2 are deduced as:

$\gamma\left(\frac{1}{R_{1}}+\frac{\sin \phi}{x_{1}}\right)=\frac{2 \gamma}{R_{0}}+(\Delta \rho) g z_{1}$ 


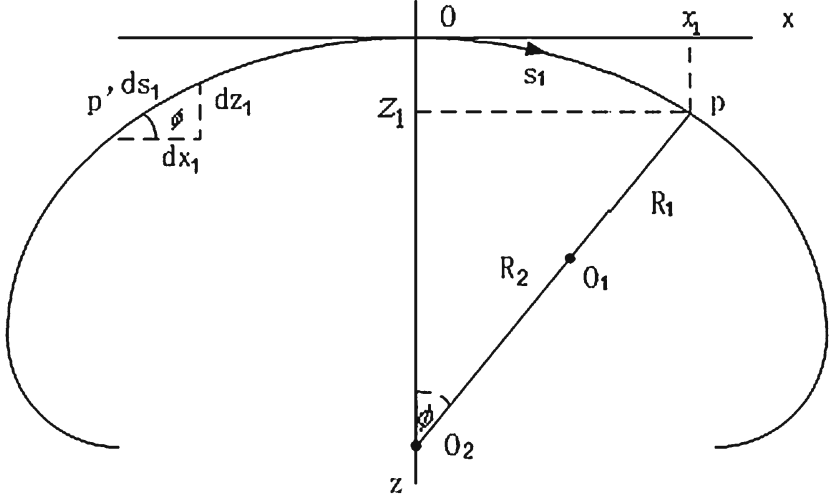

Fig. 4 Geometry analysis graph of the droplet (Yuan et al. 2002)

In which, $\phi$ is the rotate angle between the tangent line at $p$ point and the data plane. Equation 3 is the theoretical base to calculate surface tension.

Because of the symmetry properties, only the right half curve is to be considered, the left half curve is obtained by mirroring. So the right half curve is expressed simply as: $x_{1}=x_{1}\left(s_{1}\right)$ and $z_{1}=z_{1}\left(s_{1}\right)$, in which $s_{1}$ is the length of arc from origin to the point.

From Fig. 4, we can get: $d x_{1} / d s_{1}=\cos \phi, d z_{1} / d s_{1}=$ $\sin \phi$, because $d s_{1}=R_{1} d \phi$, from Eq. 3, we can get:

$\frac{d \phi}{d s_{1}}=\frac{2}{R_{0}}+\frac{(\Delta \rho) g}{\gamma} z_{1}-\frac{\sin \phi}{x_{1}}$

Boundary conditions are $x_{1}(0)=z_{1}(0)=\phi(0)$.

As shown in Fig. 5, suppose that $u_{n}, n=1,2, \cdots N$ is a set of experimental points which describe the meridian section of an interface and $v=v\left(s_{1}\right)$ is a calculated Laplacian curve. The objective function is defined as:

$E=\frac{1}{2} \sum_{i=1}^{N}\left[d\left(u_{n}, v\right)\right]^{2}$.

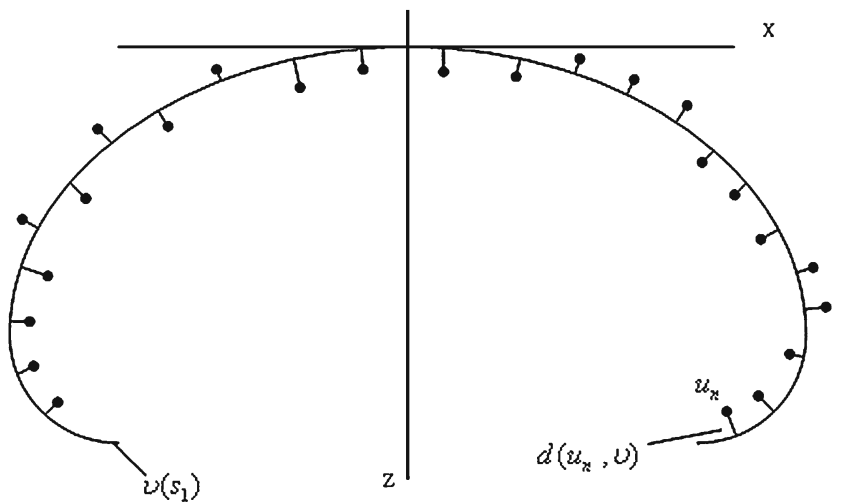

Fig. 5 Contour approach point graph
Where $d\left(u_{n}, v\right)$ is the normal distance between $u_{n}$ and the curve $v$. The value of the function will assume a minimum value corresponding to the correct shape of the curve and the correct positioning of the origin ( $\mathrm{Yu}$ and Ning 2008).

\section{Contour Fitting Method}

The origin of coordinate is determined by the intersect point of the middle axis and the first row on the contour. When origin of coordinate is determined, the coordinates of other points on contour are also determined. For the coordinates of contour points $s_{1}, x_{1}$ and $z_{1}$, they are normalized as:

$s=\frac{s_{1}}{R_{0}}, x=\frac{x_{1}}{R_{0}}, z=\frac{z_{1}}{R_{0}}$,

According to the geometry relation in Fig. 4 and taking the point of $s_{1}, x_{1}$ and $z_{1}$ into formula (4) to get the following equation group:

$\frac{d x}{d s}=\cos \phi$,

$\frac{d z}{d s}=\sin \phi$

$\frac{d \phi}{d s}=2+\frac{(\Delta \rho) g R_{0}^{2}}{\gamma} z-\frac{\sin \phi}{x}=2+\beta z-\frac{\sin \phi}{x}$

In Eq. $6, \beta=(\Delta \rho) g R_{0}^{2} / \gamma$. $\beta$ is the only unknown parameter, so $\beta$ is as the shape parameter of contour curve.

For every $\beta$, we can get a set of fitting results $\left(x_{i}, z_{i}\right)$ and $\phi_{i}, i=1,2, \cdots N$ by solving the Eq. 6 using RungeKutta method. We assume experiment profile point is $\left(X_{i}, Z_{i}\right) i=1,2, \cdots N$, then the error function is expressed as:

$E=\sum_{i=1}^{N} e_{i}$

In which, $e_{i}=\frac{1}{2}\left[\left(R_{0} x_{i}-X_{i}\right)^{2}+\left(R_{0} z_{i}-Z_{i}\right)^{2}\right]$.

Choose $R_{0}$ and $\beta$ to make $E$ the least. Then $R_{0}$ and $\beta$ are determined. For $\beta=(\Delta \rho) g R_{0}^{2} / \gamma$, the surface tension can be expressed as:

$\gamma=(\Delta \rho) g R_{0}^{2} / \beta$

Because $\Delta \rho, g, R_{0}^{2}$ and $\beta$ all have determined values, the surface tension $\gamma$ can be calculated.

When the correct calculated Laplacian curve $v$ is determined, the contact angle is determined: it is the angle $\phi_{i}$ at the point $\left(x_{i}, z_{i}\right)$ where the liquid interface meets the solid surface. 


\section{Calculation of Evaporation Velocity}

Through the numerical integration method, volume and surface area of droplet can be calculated in accordance with the contour of droplet. As shown in Fig. 6, by accumulating volumes and lateral areas of all units, droplet size and surface area can be obtained.

With the droplet contour, the diameter of cross section $D_{i},(i=1,2, \cdots N)$ in pixel can be determined by difference between the position of extreme right and extreme left in every line. According to numerical integration, the surface area of droplet $S$ is expressed as:

$S=\sum_{i=1}^{N} S_{i}=\pi \sum_{i=1}^{N} l_{i}\left(r_{i}+r_{i+1}\right)=\frac{1}{2} \pi \sum_{i=1}^{N} l_{i}\left(D_{i}+D_{i+1}\right)$

In which, $S_{i}$ is the lateral area of integral unit (frustum of cone), generatrix of integral unit $l_{i}=$ $\sqrt{\left(r_{i}-r_{i+1}\right)^{2}+\Delta H^{2}}, \Delta H=1$.

Similarly, the volume of droplets $V$ can be calculated as follow:

$V=\sum_{i=1}^{N} V_{i}=\frac{\pi}{4} \sum_{i=1}^{N} D_{i}{ }^{2} \Delta H$

In which, $V_{i}$ is the volume of integral unit.

Using formula (9) and (10), we can calculate the surface area and the volume of droplet $\left(S_{1}, V_{1}\right)$ at time $t$. After time $\Delta t$, we can get the image of droplet again and can calculate the surface area and volume of the droplet $\left(S_{2}, V_{2}\right)$ at that time. Thus, we can get the evaporation velocity of droplet within the period of time:

$v=\frac{\Delta V \rho}{\Delta t S_{\text {mean }}}=\frac{\left(V_{1}+V_{i n j}-V_{2}\right) \rho}{\Delta t\left(S_{1}+S_{2}\right) / 2}$

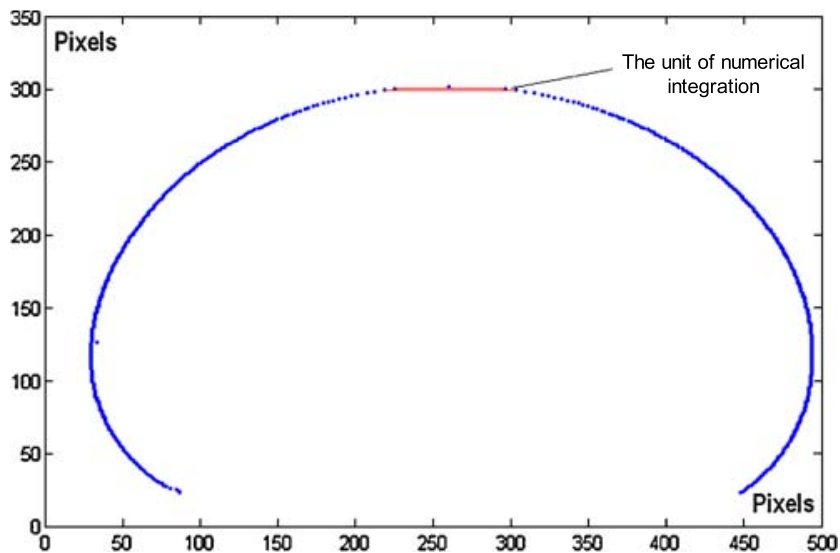

Fig. 6 Schematic diagram of numerical integration

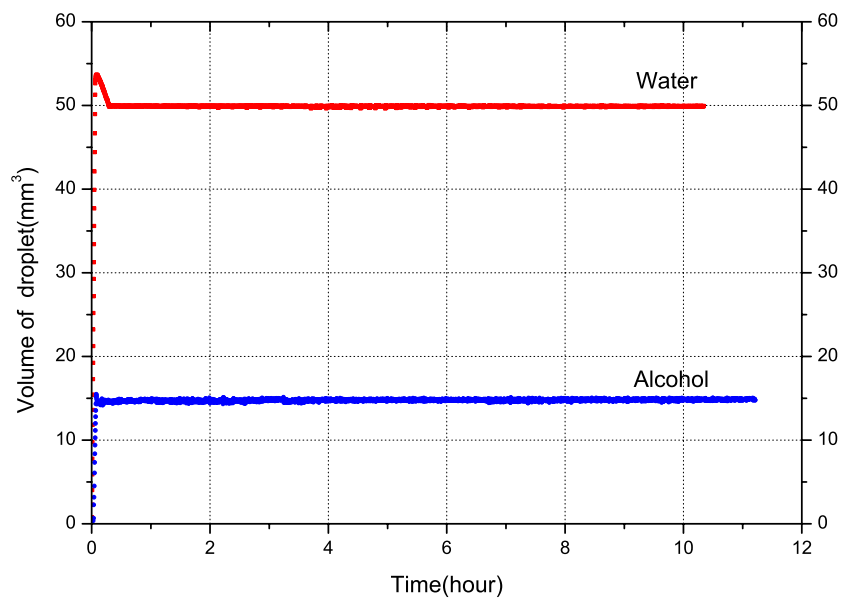

Fig. 7 Volume fluctuations of water and alcohol droplet during control process

In which, $S_{\text {mean }}$ is the average surface area in $\Delta t, V_{i n j}$ is the injection volume in $\Delta t$.

\section{Experimental Results}

In order to verify the effect of the system, we conduct the experiments with water droplet and alcohol droplet respectively. The following pictures show the experimental results (Figs. 7 and 8).

According to the experimental results, the fluctuation of the volume of water droplet is less than $0.5 \mathrm{~mm}^{3}$. The relative fluctuation rate is less than $1 \%$. Meanwhile, the fluctuation of the volume of alcohol droplet is less than $0.8 \mathrm{~mm}^{3}$, and the relative fluctuation rate is less than $5.3 \%$. The fluctuation volume difference

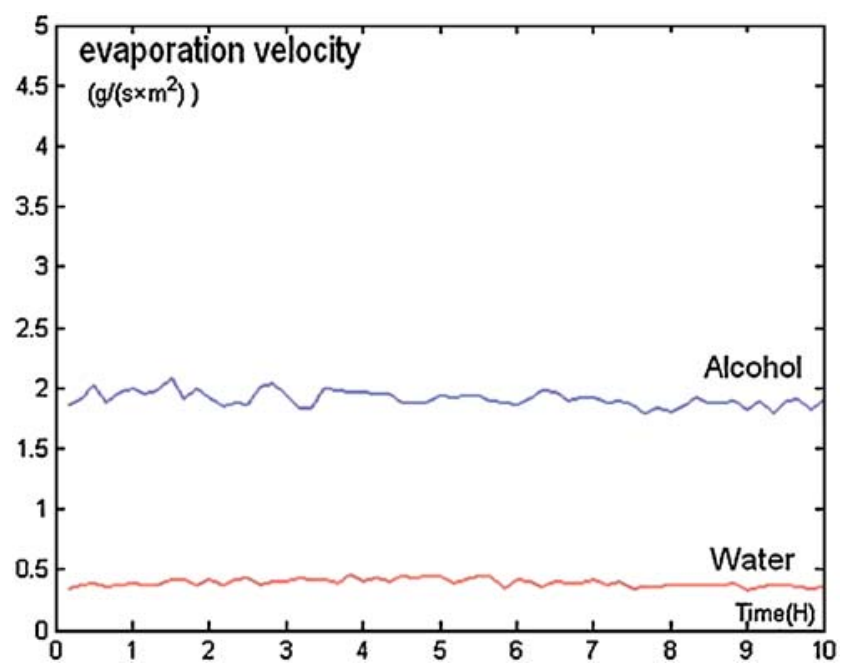

Fig. 8 Evaporation velocity of water and alcohol droplet 
between the two liquids is very small. However, the relative fluctuation rate of alcohol is relatively higher, because the evaporation velocity of alcohol is faster, and the target volume of alcohol is smaller than that of water.

\section{Conclusion}

The droplet image feedback control system can maintain the volume of droplet constant in a precision of better than $5.3 \%$, which can satisfy the needs of the steady-state evaporation space experiments planned on future Chinese satellite. Furthermore, it can obtain the parameters of droplet, such as volume, surface area, surface tension and evaporation velocity.

The function of the system can be improved in following aspects: (1) The current control cycle is within 10 seconds. By reducing the DSP processing time, we can make the control cycle shorter than before. This will improve the precision of controlling. (2) By improving control methods and adjusting the control parameters, we can achieve a better control effect.

\section{References}

Atae-Allah, C., Cabrerizo-vilchez, M., Gomez-Lopera, J.F., et al.: Measurement of surface tension and contact angle using entropic edge detection. Meas. Sci. Technol. 12, 288-298 (2001)

Badam, V.K., Kumar, V., Durst, F., Danov, K.: Experimental and theoretical investigations on interfacial temperature jumps dring evaporation. Exp. Therm. Fluid Sci. 32(1), 276-292 (2007)

Bechstedt, F.: Principles of Surface Physics. Springer, Berlin (2003)
Cai, S.J., Yu, Q.: Optimization and application of connected component labeling algorithm based on run-length encoding. J. Comput. Appl. 28(12), 3150-3153 (2008)

Duan, F., Badam, V.K., Durst, F., Ward, C.A.: Thermocapillary transport of energy during water evaporation. Phys. Rev. E 72(5), 056303(1-11) (2005)

Erbil, H.Y.: Surface Chemistry of Solid and Liquid Interfaces. Blackwell, Oxford (2006)

Gonzalez, R.C., Woods, R.E., Eddins, S.L.: Digital Image Processing Using MATLAB. Prentice-Hall, Upper Saddle River (2003)

Hartland, S.: Surface and Interfacial Tension: Measurement, Theory, and Applications. CRC, Boca Raton (2004)

Hoorfar, M., Neumann, A.W.: Axisymmetric drop shape analysis (adsa) for the determination of surface tension and contact angle. J. Adhesion 80, 727-743 (2004)

Ning, Q., Zhu, Z.Q.: Determine the surface tension and contact angle of drop by image processing method. Chinese J. Space Sci. 28(1) (2008)

Popov, S., Melling, A., Durst, F., Ward, C.A.: Apparatus for investigation of evaporation at free liquid-vapour interfaces. Int. J. Heat Mass Transfer 48, 2299-2309 (2005)

Rotenberg, Y., Boruvka, L.: Determination of surface tension and contact angle from the shapes of axisymmetric fluid interface. J. Colloid Interface Sci. 93(1), 169-182 (1983)

Suzuki, K., Horiba, I., Sugie, N.: Linear-time connectedcomponent labeling based on sequential local operations. Comput. Vis. Image Underst. 89(1), 1-23 (2003)

Weinberger, M.J., Seroussi, G., Sapiro, G.: The LOCO-I Lossless image compression algorithm: principles andstandardization into JPEG-LS. IEEE Trans. Image Proc. 9(8), 1309-1324 (2000)

Weszka, J.S.: A survey of threshold selection techniques. Comput. Graphics Image Proc. 7, 259-265 (1978)

$\mathrm{Yu}$, Q., Ning, Q.: Image feedback control system in the space drop evaporation experiment. Chinese J. Space Sci. 28(1) (2008)

Yuan, Z.F., Mukai, K., Takagi, K., Ohtakam, M., Huang, W.L., Liu, Q.S.: Surface tension and its temperature coefficient of molten tin determined with the sessile drop mETHOD at different oxygen partial pressures. J. Colloid Interface Sci. 254(2), 338-345 (2002)

Zhu, Z.Q.: Experimental investigation on the coupling mechanism of thermocapillary convection and evaportaion ffect. $\mathrm{Ph}$. D. thesis, Institute of Mechanics, Graduate School of Chinese Academy of Sciences (2009) 